\title{
A fiber based synchronously pumped tunable Raman laser in the NIR
}

\author{
Shaif-ul Alam, Dejiao Lin, Peh Siong Teh, Kang Kang Chen and David J. Richardson \\ Optoelectronics Research Centre, University of Southampton, Southampton, SO17 1BJ, United Kingdom
}

Raman lasers have attracted much interest since they allow a very wide range of wavelengths to be generated [1]. Operation in the pulsed regime is compromised by the power dependence of the gain - different parts of the pulse with different instantaneous power will undergo differing amounts of Raman scattering leading to major variations in spectral content across the pulse (varying amounts of light in different Raman orders). To address this issue active pulse shaping technique can be applied to obtain rectangular shaped output pulses so that constant Raman gain can be ensured across the pulse profile [2]. Here we demonstrate a new approach combining pulse shaping with a synchronously pumped scheme [3] to produce narrower linewidths as well as higher extinction ratios between neighboring Raman Stokes lines. Several tens of nanometer of tuning range for each Stokes order and a total tuning range of over $200 \mathrm{~nm}$ was achieved using resonant feedback from an external bulk grating.
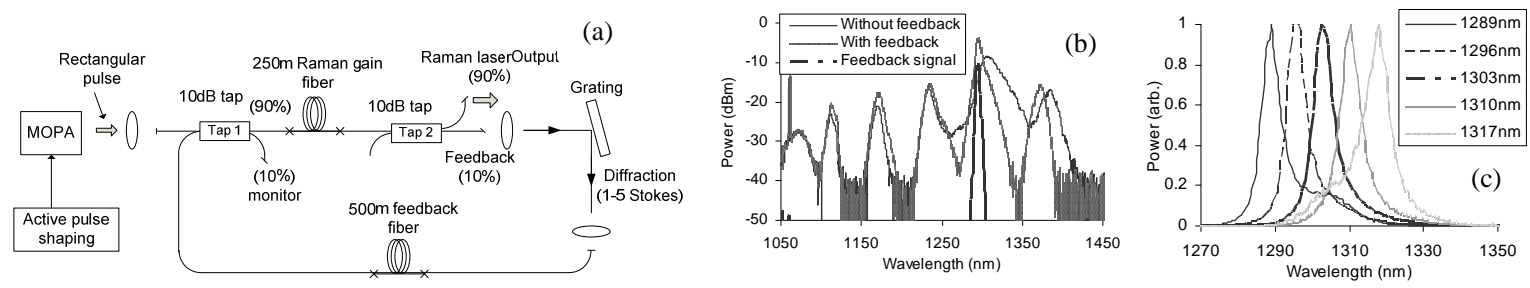

Fig. 1 (a) The layout of the Raman fiber laser synchronously pumped by a PM fiber MOPA operating at $1060 \mathrm{~nm}$; (b) Spectra of the $4^{\text {th }}$ Raman Stokes line with and without feedback; (c) Spectra of the tunable Raman laser at $1.3 \mu \mathrm{m}$ corresponding to the $4^{\text {th }}$ Stokes order.

A schematic of the synchronously pumped near-infrared (NIR) Raman laser is illustrated in Fig. 1(a). The MOPA was seeded by a $1060 \mathrm{~nm}$ fiber Bragg grating stabilized semiconductor laser diode which passed through an in-line electro-optic modulator (EOM), driven by an arbitrary waveform generator (AWG), for active pulse shaping. The seed signal was amplified by an all-fiber, three-stage, Yb-doped PM amplifier chain to deliver single mode, single polarization output with the required rectangular pulse shape. A 250m long Freelight fiber was used as the Raman gain medium. A second length of 500m fiber was used to synchronize the pump and the feedback signals.

We investigated the synchronously pumped Raman Stokes starting from the $1^{\text {st }}$ order up to the $4^{\text {th }}$ order. Fig. 1(b) plots example spectra for the $4^{\text {th }}$ order Raman Stokes. The pulse width was 16 ns and the repetition rate was set at $400.3 \mathrm{kHz}$ to meet the need for synchronization. As shown in Fig. 1(b), even though the feedback signal power was less than $0.1 \%$ of the pump power, substantial improvement in terms of the linewidth and extinction ratio of the $4^{\text {th }}$ order Raman Stokes was observed by comparing the spectra with and without the feedback signal (centered at $1303 \mathrm{~nm}$ ). Due to the broad Raman gain bandwidth, the output of the Raman laser can be tuned by adjusting the wavelength of the feedback signal using an external bulk grating along the feedback path. As shown in Fig. 1(c), the continuous tuning range of the $4^{\text {th }}$ Stokes was about $28 \mathrm{~nm}$ equivalent to $5.0 \mathrm{THz}$ in the frequency domain. The peak powers of the MOPA and Raman laser outputs (from the $90 \%$ port of Tap 2) were $281 \mathrm{~W}$ and $119 \mathrm{~W}$ respectively, which was reasonable considering the fiber launch losses and fiber attenuation at $1.0 \mu \mathrm{m}$. Further enhancement in output power is possible by shortening the Raman gain medium at a fixed pulse duration and repetition rate to increase the peak power required to achieve energy transfer to a given Raman order. We also studied the laser performance for other Raman Stokes order and their detailed performance characteristics will be presented at the conference.

In conclusion, we have demonstrated a synchronously pumped Raman laser source in the NIR region, pumped by a single polarization all-fiber Yb-doped PM MOPA incorporating adaptive pulse shaping. A tuning range up to $5 \mathrm{THz}$ was achieved within each Stokes orders by means of an external bulk grating. Incorporation of a tunable fiber Bragg grating (FBG) should enable an all-fiber tunable pulsed Raman laser.

\section{References}

[1] Erik Bélanger, Martin Bernier, Dominic Faucher, Daniel Côté, and Réal Vallée, "High-Power and Widely Tunable All-Fiber Raman Laser," J. Lightwave Technol. 26, 1696-1701 (2008).

[2] K.K. Chen, S. U. Alam, P. Horak, C.A. Codemard, A. Malinowsky, D.J. Richardson, "Excitation of individual Raman Stokes lines in the visible regime using rectangular-shaped nanosecond optical pulses at 530 nm”, Opt. Lett., 35, 2433 (2010)

[3] K. Smith, P. N. Kean, D. W. Crust, and W. Sibbett, "An Experimental Study of a Synchronously Pumped Fibre Raman Oscillator ", J. Mod. Opt. 34, 1227 (1987). 